\title{
Real Time Object Identification for Intelligent Video Surveillance Applications
}

\author{
L.Menaka \\ R.V.S.College of Engineering \\ and Technology \\ Karaikal, India
}

\author{
M.Kalaiselvi Geetha \\ Annamalai University \\ Chidambaram, India
}

\author{
M.Chitra \\ R.V.S.College of Engineering \\ and Technology \\ Karaikal, India
}

\begin{abstract}
Intelligent video surveillance system has emerged as a very important research topic in the computer vision field in the recent years. It is well suited for a broad range of applications such as to monitor activities at traffic intersections for detecting congestions and predict the traffic flow. Object classification in the field of video surveillance is a key component of smart surveillance software. Two robust methodology and algorithms adopted for people and object classification for automated surveillance systems is proposed in this paper. First method uses background subtraction model for detecting the object motion. The background subtraction and image segmentation based on morphological transformation for tracking and object classification on highways is proposed. This algorithm uses erosion followed by dilation on various frames. Proposed algorithm in first method, segments the image by preserving important edges which improves the adaptive background mixture model and makes the system learn faster and more accurately. The system used in second method adopts the object detection method without background subtraction because of the static object detection. Segmentation is done by the bounding box registration technique. Then the classification is done with the multiclass SVM using the edge histogram as features. The edge histograms are calculated for various bin values in different environment. The result obtained demonstrates the effectiveness of the proposed approach.
\end{abstract}

Keywords: object classification, SVM, morphological operator, edge histogram

\section{INTRODUCTION}

In the present scenario, a large amount of security measures are needed in public areas due to terrorist activities. Manual surveillance is highly impractical. Hence automated video surveillance becomes essential. In that rationale, object identification is the preliminary step. The ability to reliably detect pedestrians from video data has very important applications in many fields like, intelligent transportation, automated surveillance, security, robotics and assistive technology for visually impaired, advanced human machine interfaces, automated driver assistance systems in vehicles etc.

Pedestrian are more vulnerable to accidents and collisions involving pedestrians often produce severe injuries. Accurately detecting pedestrians from a video is one of the most challenging tasks for object detection which attracts most of the researchers working in this field. This paper proposes an approach for conditions both pedestrian, vehicle and tree identification in real time with and without background subtraction technique.

\subsection{Difficulties in object identification}

The main challenges of vision-based object identification for automated video surveillance applications are listed below

\subsubsection{The image background may be cluttered with other stationary object:}

This is especially obvious for the urban road scenes. This may complicate the object identification in the video surveillance system.

\subsubsection{Images are captured from a moving platform:}

The captured images may contain vehicles and other objects at different distances, in a background that constantly changing, when the test vehicle is moving. This makes the background subtraction technique commonly used to detect objects in a video surveillance system unsuitable for this usage.

\subsubsection{Variable outdoor illumination conditions:}

The illumination of the road environment may change during different times of day and under different weather conditions. This may affect the quality of the captured images.

\subsubsection{Occlusion and different poses of objects in a} image:

In moving object identification, different poses of pedestrian and vehicle need to be considered for the accurate identification. Consideration of different poses of human and vehicles are difficult in the implementation of video surveillance system. The occlusion is also unavoidable in the case of traffic congestion.

\section{RELATED WORK}

Vision based vehicle detection is an area of research in the intelligent transportation systems community. In the literature, many studies have been performed on the static images. A support vector machine(SVM) approach was used in [1], built multiple detectors using Haar wavelets, PCA, truncated wavelet features using neural networks and SVM classifiers. Template matching is one of the methods used for vehicle detection and tracking. In this method, Scale Invariant Feature Transform (SIFT) points were extracted which is invariant to image scaling and rotation and partially invariant to change in illumination and 3D rotation. A statistical approach has been used in [3], performing vehicle detection using principle component analysis (PCA) and independent component analysis(ICA) to do classification on a statistical model and increased its speed by modeling the PCA and ICA vectors with the weighted Gaussian mixture model. A review of ecent template matching methods for detection and tracking of vehicle is presented in [4]. The binary classification scheme is 
an efficient tool which can be used for object detection and matching which is described in [7].

There are many technologies that are currently being used for pedestrian and vehicle detection such as ultrasonic sensors, Doppler radar sensors piezo-metric sensors etc which is presented in [8]. These sensors while being very effective have various drawbacks ranging from cost effectiveness to durability. The "irregular blobs" can be detected and then cluster the pieces according to the common motion constraint of the extracted features. It reduces the computational costs by limiting the feature analysis only to "irregular blobs" is presented in [9]. Using 2D and 3D models for detection has also been explored by several authors. A 3D model-based detection approach with background subtraction is presented in [11]. The 2D templates has been created from the 3D vehicle models which is used to generate multiple hypotheses for a given foreground mask. This approach use only the template contours, so like the feature based approaches, its performance on noisy, low resolution and crowded scenes is uncertain.

The partial occlusion problem is addressed by a feature based tracking algorithm in [14]. The detection is done based on the "corner" features and then grouped them according to a "common motion constraint". However, both algorithms depend purely on the accuracy of feature detection and matching, which makes them error prone in noisy, low resolution videos. Common motion constraint is not applicable in very crowded scenes, where the vehicles are forced to move at similar speeds. Video based detection emerged as an important aspect of research, as proliferation high performance cameras and faster inexpensive computing systems became assessable.

This paper introduces two methods at detection stage. First method for classifies the objects using height and width. Second method classifies the object by Support Vector Machine (SVM) classifier. The method combines edge histogram and SVM. The training of the SVM classifier is carried out offline.

The proposed classification procedure is useful not only for increasing the speed and reliability of feature matching but also for reducing the computational load associated with the robust estimation of the registration parameters.

\section{HEIGHT - WIDTH BASED CLASSIFICATION}

\subsection{BACKGROUND SUBTRACTION}

The main reason of using background subtraction is that it works well if the background is static for long time. In traffic surveillance system, camera often remains static. This approach uses mixture of Gaussian for background subtraction.

The proposed approach uses height-width based classification method as seen in Fig. 1.

\subsubsection{Mixture of Gaussians}

Mixture of Gaussians method maintains a density function for every pixel and is capable of handling multi model backgrounds as in Eq. (1)

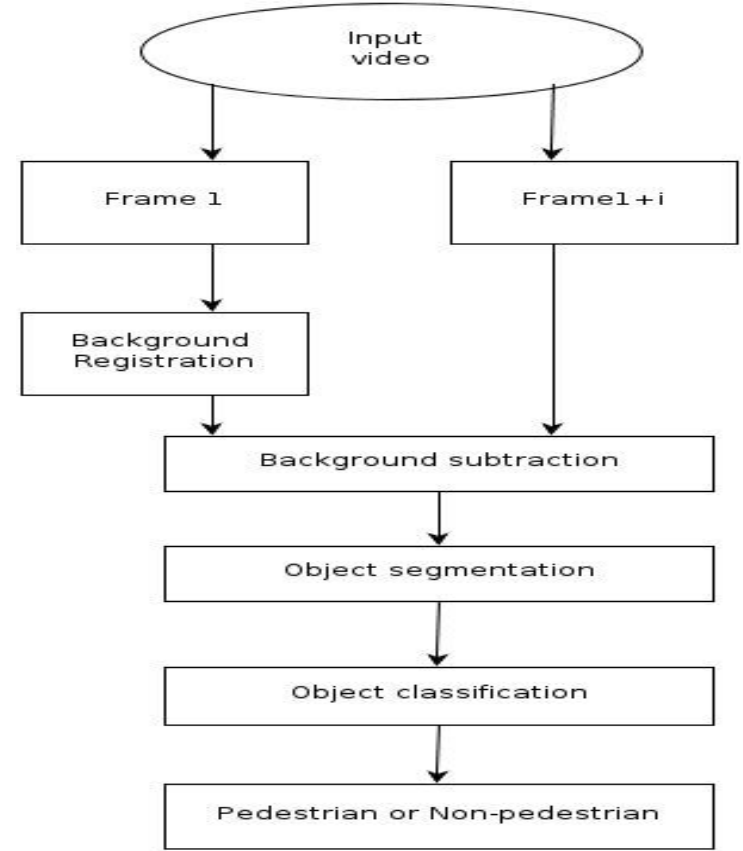

Fig. 1. A model for height-width based approach

Objects must be initially classified as pedestrians or vehicles.

$\mathrm{p}(\mathrm{x} \mid \pi, \mu, \Sigma)=\Sigma Y_{i=1} \pi_{R} N\left(x \mid \mu_{\mathrm{k}} \Sigma_{\mathrm{k}}\right)$

Where, $\mathrm{x}$ random variable

$\pi_{k}$ Mixing coe $\square$ cient of the $k^{\text {th }}$ Gaussian

$\mu_{k \text { Mean }}$ of the $k^{\text {th }}$ Gaussian

$\Sigma_{\mathrm{k}}$ Covariance of the $k^{\text {th }}$ Gaussian

Mixture of Gaussians robustly deal with lighting changes, repetitive motions, clutter, introducing or removing objects from the scene and slowly moving objects. It can be updated without having to store large number of frames in buffer hence reducing memory costs. The learning rate is passed into MOG model. The learning rate is the rate at which the model adapts to changes in the video image. Low values correspond to a slowly adapting model. High values make the model adapt quickly to scene changes. An example of Background subtraction is shown in Fig. 2(a) and Fig. 2(b).

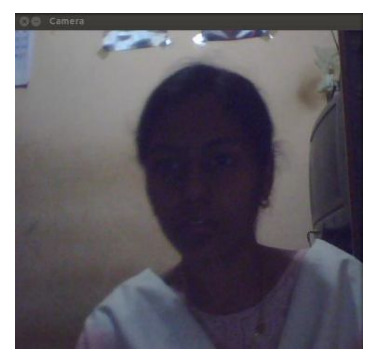

Fig. 2 (a). Input frame

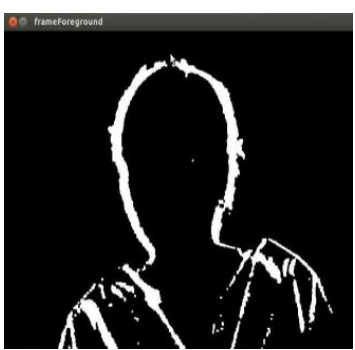

Fig. 2(b). Background subtracted frame 


\subsection{OBJECT DETECTION}

In the proposed approach shown in Fig. 2, the first frame is considered as background. The mixture of Gaussian method is used in background subtraction. Next the background is eliminated and the foreground objects only maintained. At segmentation stage, morphological operators are used. Dilation and erosion are the two morphological operators used for segmenting the object edges here.

Mathematical morphology is used for analyzing object shape characteristics such as size and connectivity, which are not easily accessed by linear approaches. After background subtraction successfully extract out the foreground, it reproduces them into a binary image as seen in Fig. 2(b). To make the objects more recognizable and informative, these objects should be marked and recorded. Contouring is the process of marking out the edge of the object, making it more recognizable and informative. Once background subtraction is done, moving objects are marked using contours. An example of object detection is shown in Fig. 3.

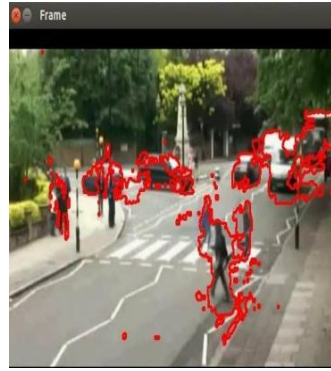

(a)Frame 123

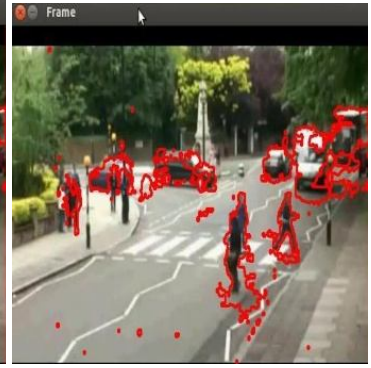

(b)Frame 125
Fig. 3. Moving object such as pedestrian and vehicle detection

\subsection{OBJECT IDENTIFICATION}

In computer vision field, object's edge is usually identified as the area of pixels that contrasts with its neighbor pixels or area of moving pixels. After getting the edge of the object, the height of the object can be measured by subtracting the top left pixel position of the rectangle and bottom left pixel position of the rectangle. The width of the object can be measured by subtracting the top left pixel position of the rectangle and top right pixel position of the rectangle. The threshold value set to 1.126 or below for pedestrian and from1.126 to 2.72 for vehicle from the ground truth results. Based on threshold value for the height and width, the object is classified whether it is a pedestrian or vehicle. An example is shown in Fig. 4 (a) and Fig. 4 (b).

Here, some of human are not boxed. These humans are standing for a long time. So these humans are considered as background using the background updating model which is explained in Section 3.

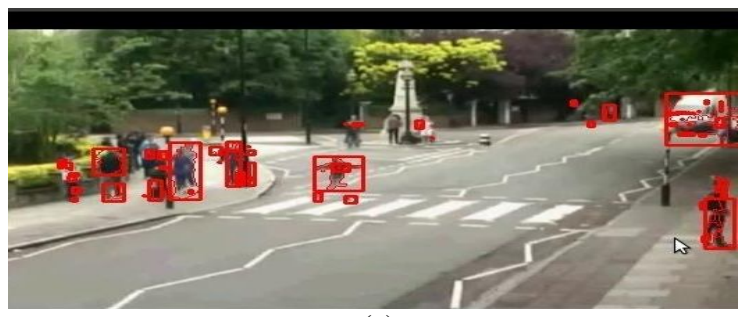

(a) 
ambient lighting. The various frames for object detection is shown in Fig. 6.

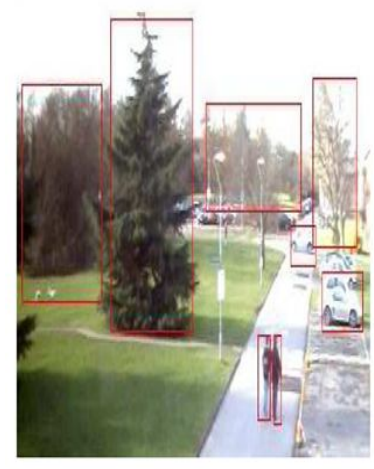

Frame 120

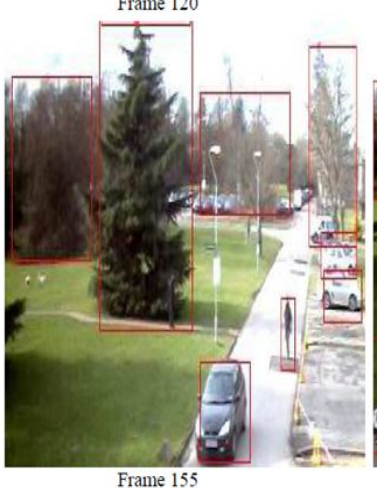

Frame 155

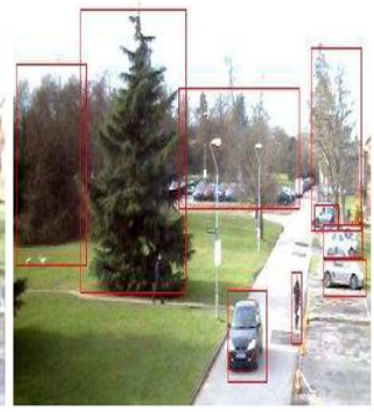

Frame 150

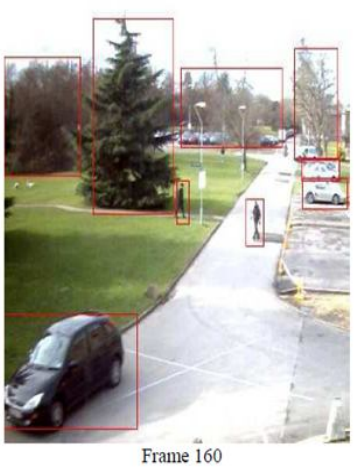

Fig. 6. Static and dynamic object detection from PETS dataset

\subsection{ROI EXTRACTION}

In this project, the next step is to develop a segmentation strategy to extract the regions of interest (ROI) corresponding to pedestrians, vehicles and trees in the images. This process is called as "bounding box registration", since the ROI is often represented by a bounding box in each video frame. A simple connected-components algorithm is used to label regions in the surveillance video. Each such region

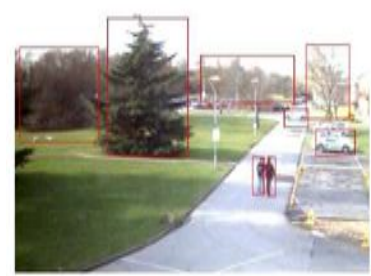

Frame 45

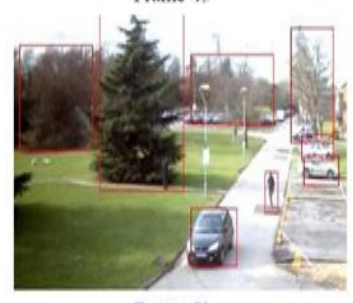

Frame 52

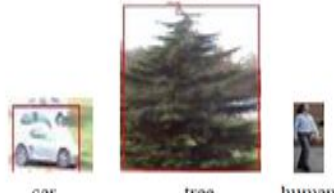

tree

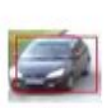

car

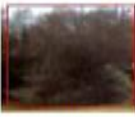

tree
Fig. 7. ROI extraction of car, tree and human

potentially includes both moving and unmoving object. Since, the classification operates on rectangular regions of pixels based on the calculated height and width of the rectangle. The bounding boxes smaller than a given threshold ( height $<42$ and width $<22$ in this experiment) is discarded which as too small to contain any of the target objects.

The OpenCV library function cvFindContours is used for connected-component analysis. For speeding up the tracking process, the region of interest (ROI) is rectangular and corresponds to the bounding box of the object. The example shows that the extracted ROI of pedestrian, vehicle and trees in Fig. 7. The frames 45 and 52 in Fig. 7 are taken from the PETS data set.

\subsection{EDGE HISTOGRAM CALCULATION}

In this approach, an object view is represented by a discrete set of points sampled regularly along the internal or external contours. For every point, histogram is computed that approximates the distribution of adjacent point locations relative to the reference point. The feature vector can be constructed from edge histogram values of the object. The histograms vectors used are therefore able to capture similarity within a class and at the same time discriminate between different classes.

There is no "best" number of bins, and different bin sizes can reveal different features of the data. Some theoreticians have attempted to determine an optimal number of bins, but these methods generally make strong assumptions about the shape of the distribution. Depending on the actual data distribution and the goals of the analysis, different bin widths may be appropriate. Experimentation is usually needed to determine appropriate bins. In this work, for the same contour edges, various histogram bins such as $6,16,32$ and 64 are used to calculate the histogram values. The output for these 4 histogram bin values are analyzed and tabulated in Section 5 .

\subsection{SUPPORT VECTOR MACHINES (SVM)}

SVM is based on the principle of structural risk minimization. For linearly separable data, SVM finds the separating hyper plane which separates the data within the largest margin. For, linearly inseparable data, it maps the data in the input space into high dimension space $\mathrm{x} \in \mathbb{R}^{\mathrm{I}} \mapsto \Phi(\mathrm{x}) \in \mathbb{R}^{\mathrm{H}}$ with kernel function $\Phi(\mathrm{x})$, to find the separating hyper plane. SVM was originally developed for two class classification problems. The $\mathrm{N}$ class classification problem can be solved using $\mathrm{N}$ SVMs. Each SVM separates a single class from all the remaining classes (One-vs.-rest approach).

Given a set of frames corresponding to $\mathrm{N}$ classes for training, $\mathrm{N}$ SVMs are trained. Each SVM is trained to distinguish a class and other classes in the training set. During testing, the class label $y$ of a class $x$ can be determined using:

$$
y=\left\{\begin{array}{l}
n, \text { if } d_{n}(x)+t>0 \\
0 . \text { if } d_{n}(x)+t<0
\end{array}\right.
$$

Where, $\operatorname{dn}(\mathrm{x})=\max \{\mathrm{di}(\mathrm{x})\} \mathrm{N} \mathrm{i}=1$, and $\mathrm{di}(\mathrm{x})$ is the distance from $x$ to the SVM hyper plane corresponding to frame $i$, the classification threshold is $\mathrm{t}$, and the class label $\mathrm{y}=0$ stands for unknown. The model for SVM based classification is given in Fig. 8. 


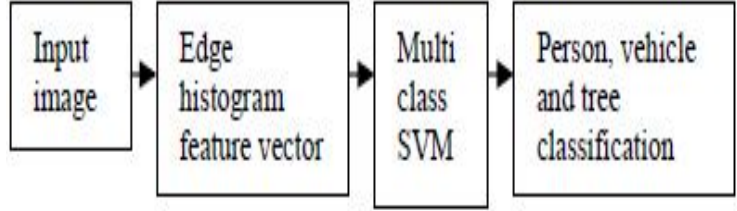

Fig. 8.Work flow of the SVM approach

The Fig. 9 addresses the key issues in the development of SVM trained pattern classifier. The representation and the learning engine. The architectural overview of the system is provided in Fig. 9 as applied to the task of people, vehicle and tree detection and shows the training and testing phases. In the training step, the system takes as input 1) a set of images of the object class that have been aligned and scaled so that they are all in approximately the same position and the same size and 2) a set of patterns that are not in our object class.

An intermediate representation that encapsulates the important information of three object class is computed for each of these patterns, yielding a set of feature vectors. These feature vectors are used to train a pattern classifier to differentiate between in-class and out-of-class patterns. In the testing phase, detecting objects in out-of-sample images are also considered.

\section{EXPERIMENTAL RESULTS}

\subsection{EXPERIMENTAL SETUP}

Exhaustive experiments are conducted to evaluate the performance of the proposed approach. Experiments were conducted on Intel Pentium V processor with $2.67 \mathrm{GHz}$ speed with 2GB RAM and the object identification was done in C++ with OpenCV library.

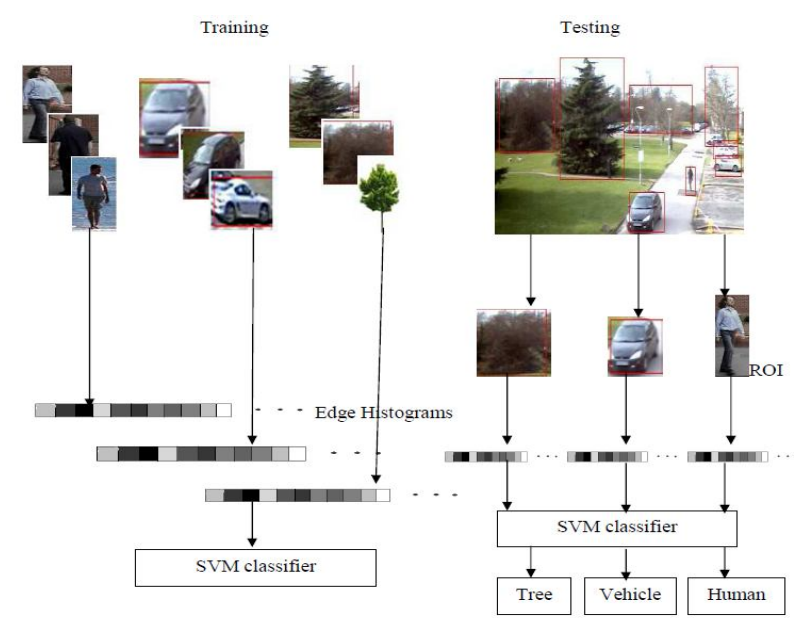

Fig. 9. Training and Testing of SVM classifier

\subsection{EXPERIMENTAL DATASETS}

To examine the performance of the proposed method, four video set was collected from a PETS dataset is shown in table 1. The video data is split into frames at 20 frames per second with OpenCV library and sampled at a resolution of $320 \mathrm{X}$ 240. The sequences of objects were automatically extracted using background subtraction method and ROI registration method.

\begin{tabular}{|l|l|l|l|l|}
\hline \multicolumn{1}{|c|}{ Video set } & $\begin{array}{c}\text { Duration } \\
(\mathrm{sec})\end{array}$ & \multicolumn{1}{|c|}{$\begin{array}{c}\text { Human } \\
(\mathrm{sec})\end{array}$} & $\begin{array}{c}\text { Vehicle } \\
(\mathrm{sec})\end{array}$ & $\begin{array}{l}\text { Tree } \\
(\mathrm{sec})\end{array}$ \\
\hline $\begin{array}{l}\text { Pedestrian, } \\
\text { car and trees }\end{array}$ & 30 & 526 & 1121 & 387 \\
\hline $\begin{array}{l}\text { Pedestrian , } \\
\text { all type of } \\
\text { vehicles and } \\
\text { trees }\end{array}$ & 45 & 734 & 954 & 762 \\
\hline $\begin{array}{l}\text { Pedestrian , } \\
\text { all type of } \\
\text { vehicles and } \\
\text { trees }\end{array}$ & 60 & 976 & 1578 & 589 \\
\hline $\begin{array}{l}\text { Images under } \\
\text { illumination } \\
\text { changes and } \\
\text { different }\end{array}$ & 20 & 632 & 584 & 366 \\
\hline \multicolumn{4}{|l|}{ Table 1. Experimental dataset } \\
\hline
\end{tabular}

\subsection{PERFORMANCE METRICS}

\subsubsection{Performance evaluation of height-width based approach}

In this method, the sample image is first given into heightwidth based classification method. The aspect ratio is calculated based on the height and width of the bounding box which is given in the Eq. 1. After getting the aspect ratio, which is compared with the threshold. Based on the threshold value, the object is identified as a pedestrian or nonpedestrian. So, care should be taken when selecting the threshold value. The Figure 15, 16 and 17 shows some of the object identified frames.

The aspect ratio is calculated by

$$
\text { Aspect ratio }=\frac{\text { width }}{\text { height }}
$$

The moving pedestrian alone detected and identified in Fig. 10. Pedestrian which are boxed shows that they are moving in a road. The remaining pedestrians are static for a particular system. So, these pedestrians are not identified by this system. The Fig. 11 contains both the pedestrian and vehicle. The bounding box for the vehicle is considerably high than the bounding box of the pedestrian. From these variations, the classification is done. The height, width and aspect ratio values for both human and vehicle are shown in Fig. 12.

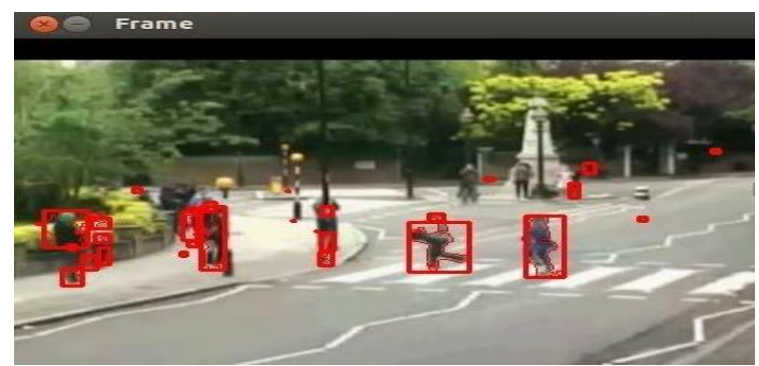

Fig. 10. Height-width based approach contains only pedestrian 


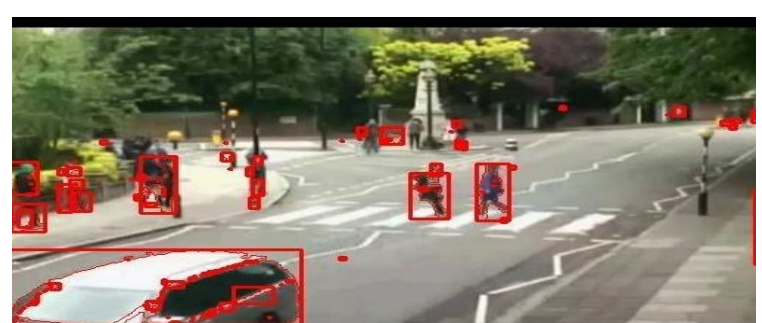

Fig. 11. Height-width based approach contains both pedestrian and vehicle which is nearer to camera

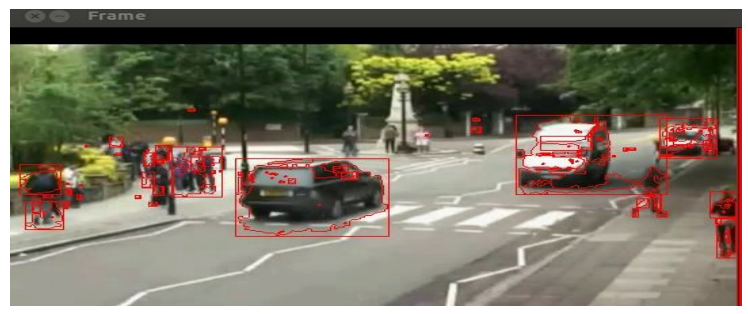

Fig. 12. Height-width based approach contains both pedestrian and vehicle which is far away from camera

This table shows the height, width and aspect ratio (calculated based on the Eq. 1). The threshold value set to below and equal to 1.126 for pedestrian and from 1.126 to 2.72 for vehicle by trial and error after conducting several experiments. The highlighted box shows the threshold values such as $1.8,1.7419,1.64255,1.81667$ and 1.5617 which values fall between 1.126 and 2.72. So these boxes are considered as vehicles. Remaining values are below the 1.126 which are categorized as pedestrian.

In this work, from the ground truth results, the performance can be evaluated by true positive rate and false positive rate. This quantity measures recall, precision and localization. Based on the equation (2) and (3) the area of the ROC curve is measured

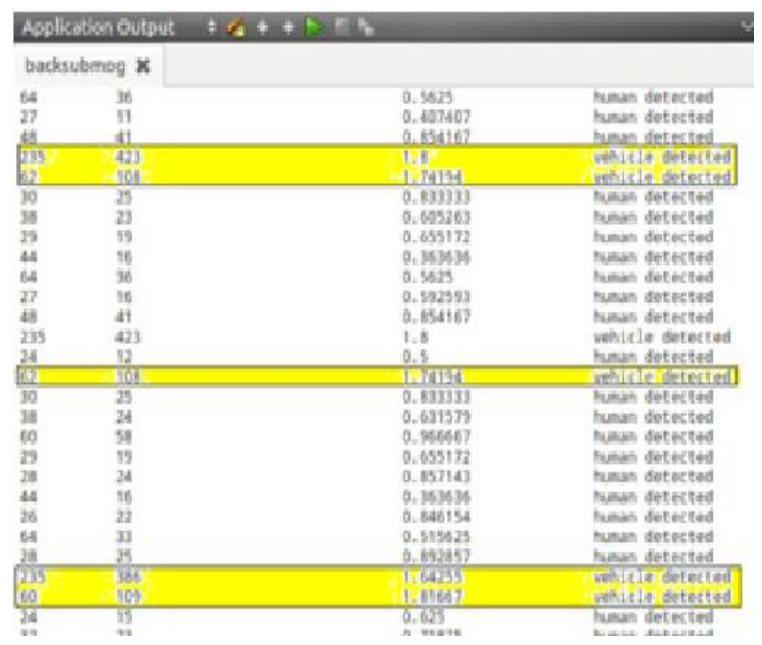

Fig. 13. Object Classification done under height-width approach

The true positive rate TPR is the percentage of non occluded objects in the camera's view that are detected. TPR is assessed by dividing the number of truly detected objects by the total number of objects.
TPR is defined by

$$
\mathrm{TPR}=\frac{\text { detected objects }}{\text { Total number of objects }}
$$

The false positive rate FPR is the proportion of detection that were not true objects. The FPR is assessed by dividing the number of false positives by the total number of detections. This is the percentage of erroneous detection. FPR is a measure of precision and localization. It is defined by

$$
\text { FPR }=\frac{\text { false positives }}{\text { detected objects }+ \text { false positives }}
$$

Table 2.Performance of height-width based approach

\begin{tabular}{|l|l|l|}
\hline $\begin{array}{l}\text { Height-width based } \\
\text { classification method }\end{array}$ & $\begin{array}{l}\text { TPR } \\
(\%)\end{array}$ & $\begin{array}{l}\text { FPR } \\
(\%)\end{array}$ \\
\hline Pedestrian only & 91.1 & 6.8 \\
\hline Both pedestrian and car & 90.9 & 7.8 \\
\hline $\begin{array}{l}\text { Both pedestrian and all } \\
\text { other types of vehicles }\end{array}$ & 91.3 & 11.2 \\
\hline
\end{tabular}

The table 2 shows the Average true positive ratio and false positive ratio for 25 frames that containing only pedestrian, 50 frames that containing both pedestrian and only car and 75 frames contain both pedestrian and all types of vehicle.

\subsubsection{PERFORMANCE EVALUATION OF STATIC- DYNAMIC OBJECT CLASSIFICATION METHOD}

The training and testing is done with the four video set represented with its duration in Table2. Three dataset is generated dynamically at the execution of each video set. Each dataset contains thousands of images. These videos are sampled at a resolution of 320X 240 at 20 frames per second. These video set contains 2034, 2450, 3143 and 1582 images respectively. The number of human, vehicle and trees are tabulated for each video set in Table 1 .

\subsubsection{Training set}

The training set consists of 10000 positive images and the class 1,2 and 3 consists of 3500,4000 and 2500 respectively. The images were originated from 3000 properly cropped object's front, rear and side view images. Since the vehicles' rear views are approximately symmetrical, the images were horizontally flipped to generate the 10000 positive training samples. The non-symmetric objects were also included in the training set to make the classifier more robust to remove any false positives picked up by the processing stage. The classifier's model created from the training is then tested on the evaluation set.

\subsubsection{Evaluation Set}

This is an independent set of 3 classes of images not used in the classifier training. They are reserved for evaluating the performance of the generated classifier and the estimation of operating points for the precision-recall plot. Video 1 contains pedestrian and vehicle of all types. This set contains 2034 positive images. Video 2 and 3 contains pedestrian, vehicle of all types and trees. This set contains 5593 positive images. Video 4 contains 1582 images under illumination changes and different poses. 


\subsubsection{PERFORMANCE OF FEATURE VECTOR USING DIFFERENT NUMBER OF HISTOGRAM BINS}

The number of histogram bins determines the fineness of the details captured by the features. Having too many bins may capture a lot of irrelevant features originated from the image's noise or the background clutter.

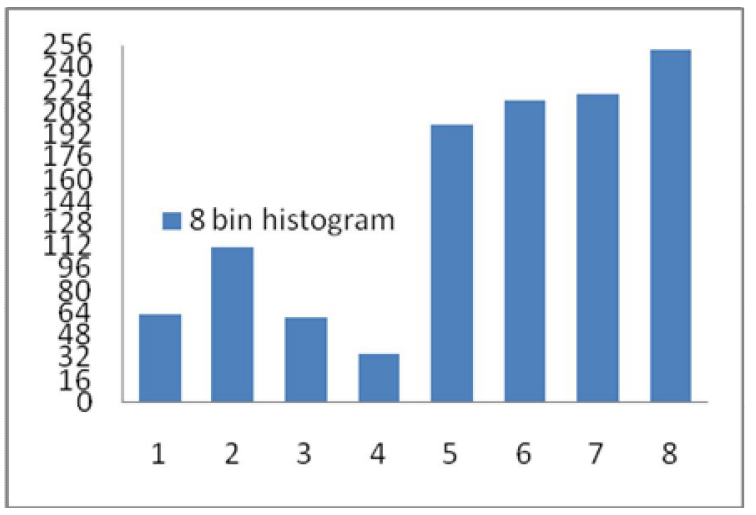

Fig. 14. 8 bin histogram

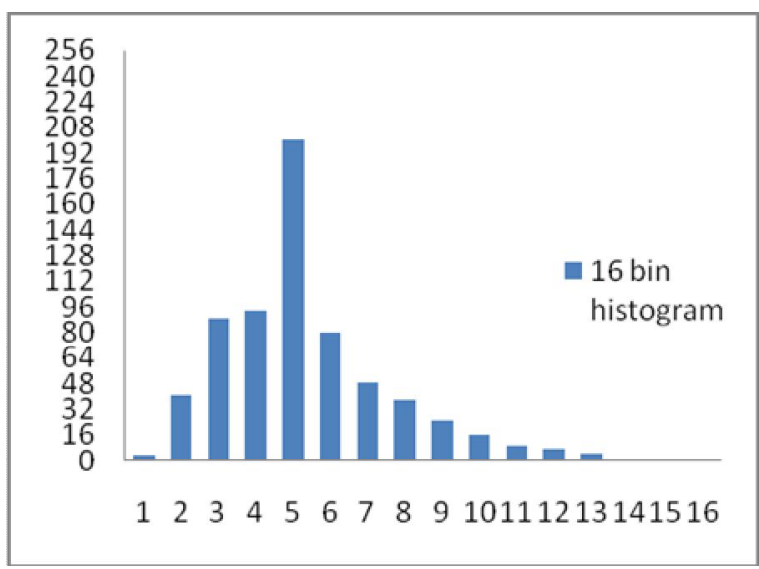

Fig. 15. 16bin histogram

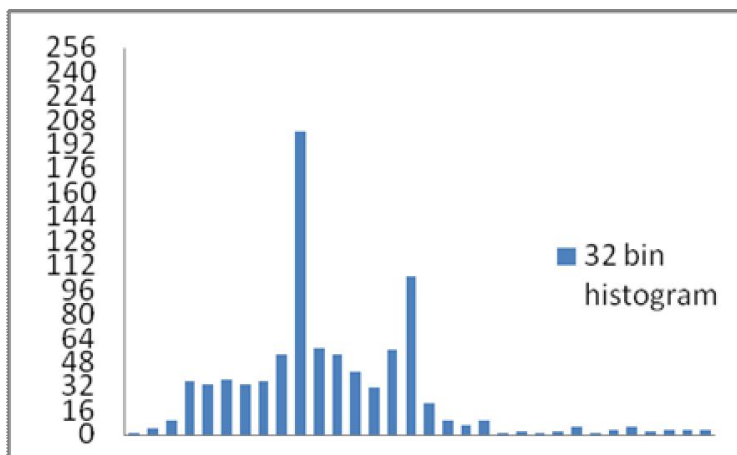

135577991113151719212325272931

Fig. 16. 32 bin histogram

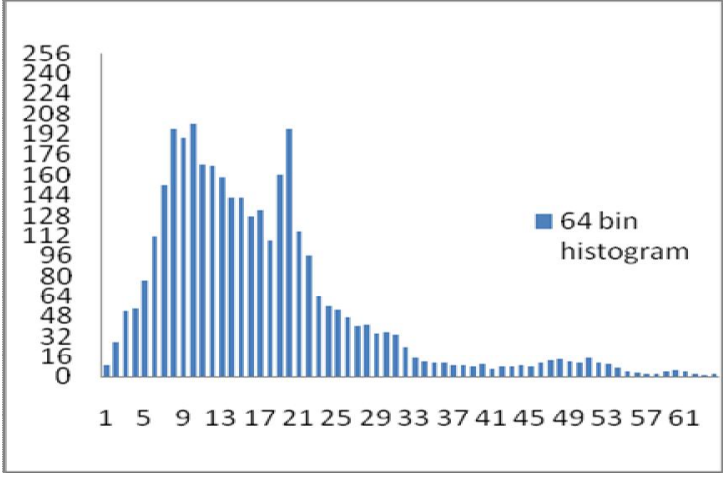

Fig. 17. 64 bin histogram

It will also slow down the classification process. On the other hand, using too few histogram bins may miss out some important object's features and thus degrade the classification performance. In this experiment, the performance of the edge features with $8,16,32$ and 64 histogram bins were evaluated which is shown in Fig. 14, Fig. 15, Fig. 16 and Fig. 17.

To evaluate the method, True positive (TP), False positive (FP) and False negative (FN) are used. In a classification task, the precision for a class is the number of true positives (i.e. the number of items correctly labeled as belonging to the positive class) divided by the total number of elements labeled as belonging to the positive class (i.e. the sum of true positives and false positives, which are items incorrectly labeled as belonging to the class). Recall in this context is defined as the number of true positives divided by the total number of elements that actually belong to the positive class (i.e. the sum of true positives and false negatives, which are items which were not labeled as belonging to the positive class but should have been).

The terms positive and negative refer to the classifier's prediction (sometimes known as the expectation), and the terms true and false refer to whether that prediction corresponds to the external judgment (sometimes known as the observation). Statistical metrics such as Precision, Recall, Accuracy and F-measure (F) gives an overall performance for this method which is explained in Equation (4), (5), (6) and (7).

Precision $(\mathrm{P})$ is the proportion of the predicted positive cases that were correct. High precision means that an algorithm returned substantially more relevant results than irrelevant.

Pracision $=\frac{x p}{x p+F}$

Recall (R) is the proportion of positive cases that were correctly identified. High recall means that an algorithm returned most of the relevant results.

$$
\text { ReCull }=\frac{T F}{T F-F N}
$$

A measure that combines precision and recall is the harmonic mean of precision and recall, the traditional F-measure or balanced F-score:

$$
F_{\mathrm{x}}=\frac{2 P R}{R-A}
$$

Accuracy (AC) is the proportion of the total number of predictions that were correct. The accuracy is the proportion of true results (both true positives and true negatives) in the population. 
International Journal of Computer Applications Technology and Research

Volume 2- Issue 6, 699 - 707, 2013, ISSN: 2319-8656

$$
\text { Acracy }=\frac{T P+T W}{T F+F F+T N+F N}
$$

The above performance metrics can be applied for the evaluation test video using different histogram bins and the performance is tabulated. Table 3 shows that the precision, recall, F-measure value and accuracy for video 1 at different histogram bin. Table 4 shows that the precision, recall, Fmeasure value and accuracy for video 2 at different histogram bin. Table 5 shows that the precision, recall, F-measure value and accuracy for video 3 at different histogram bin. Table 6 shows that the precision, recall, F-measure value and accuracy for video 3 at different histogram bin. From the values of these four tables, it can be seen that features with higher numbers of bins perform better.

Table 3. Performance of pedestrian, car and trees as seen in the dataset given in Section 6.2 for various histograms

\begin{tabular}{|l|l|l|l|l|}
\hline $\begin{array}{l}\text { Number of } \\
\text { Histogram } \\
\text { bins }\end{array}$ & $\begin{array}{l}\text { Precision } \\
(\%)\end{array}$ & $\begin{array}{l}\text { Recall } \\
(\%)\end{array}$ & $\begin{array}{l}\text { F-measure } \\
(\%)\end{array}$ & $\begin{array}{l}\text { Accuracy } \\
(\%)\end{array}$ \\
\hline 8 & 89 & 87.23 & 88.11 & 90.25 \\
\hline 16 & 90.12 & 89.12 & 89.62 & 91 \\
\hline 32 & 91.12 & 90.22 & 90.67 & 91.83 \\
\hline 64 & 91.66 & 90.76 & 91.20 & 92.93 \\
\hline
\end{tabular}

Table 4. Performance of pedestrian, all types of vehicles and trees as seen in the dataset given in Section 6.2 for various histograms

\begin{tabular}{|l|l|l|l|l|}
\hline $\begin{array}{l}\text { Number } \\
\text { of } \\
\text { Histogram } \\
\text { bins }\end{array}$ & $\begin{array}{l}\text { Precision } \\
(\%)\end{array}$ & $\begin{array}{l}\text { Recall } \\
(\%)\end{array}$ & $\begin{array}{l}\text { F-measure } \\
(\%)\end{array}$ & $\begin{array}{l}\text { Accuracy } \\
(\%)\end{array}$ \\
\hline 8 & 89.78 & 87.43 & 88.589 & 91.5 \\
\hline 16 & 93.16 & 91.25 & 92.198 & 93.12 \\
\hline 32 & 93.78 & 92.72 & 93.24 & 94 \\
\hline 64 & 95.3 & 94.1 & 94.49 & 95 \\
\hline
\end{tabular}

Table 5. Performance of pedestrian, all types of vehicles and trees as seen in the dataset given in Section 6.2 for various histograms

\begin{tabular}{|l|l|l|l|l|}
\hline $\begin{array}{l}\text { Number of } \\
\text { Histogram } \\
\text { bins }\end{array}$ & $\begin{array}{l}\text { Precision } \\
(\%)\end{array}$ & $\begin{array}{l}\text { Recall } \\
(\%)\end{array}$ & $\begin{array}{l}\text { F-measure } \\
(\%)\end{array}$ & $\begin{array}{l}\text { Accuracy } \\
(\%)\end{array}$ \\
\hline 8 & 90 & 89 & 89.49 & 92.5 \\
\hline 16 & 94 & 92 & 92.98 & 94 \\
\hline 32 & 96.7 & 92.8 & 94.71 & 94.83 \\
\hline 64 & 98 & 93.1 & 94.97 & 96 \\
\hline
\end{tabular}

Table 6. Performance of pedestrian, all types of vehicles and trees under illumination changes and different poses as seen in the dataset given in Section 6.2 for various histograms

\begin{tabular}{|l|l|l|l|l|}
\hline $\begin{array}{l}\text { Number } \\
\text { of } \\
\begin{array}{l}\text { Histogram } \\
\text { bins }\end{array}\end{array}$ & $\begin{array}{l}\text { Precision } \\
(\%)\end{array}$ & $\begin{array}{l}\text { Recall } \\
(\%)\end{array}$ & $\begin{array}{l}\text { F-measure } \\
(\%)\end{array}$ & $\begin{array}{l}\text { Accuracy } \\
(\%)\end{array}$ \\
\hline 8 & 69.78 & 68.54 & 69.15 & 67.54 \\
\hline 16 & 72.34 & 70.23 & 71.29 & 72.25 \\
\hline 32 & 75.58 & 72.65 & 74.08 & 75.25 \\
\hline 64 & 84.43 & 82.12 & 83.25 & 84.22 \\
\hline
\end{tabular}

The confusion matrix shows how the predictions are made by the model. The rows correspond to the known class of the data, i.e. the labels in the data. The columns correspond to the predictions made by the model. The value of each of element in the matrix is the number of predictions made with the class corresponding to the column for examples with the correct value as represented by the row. Thus, the diagonal elements show the number of correct classifications made for each class, and the off-diagonal elements show the errors made.

Table 7. Confusion matrix for pedestrian, car and trees as seen in the dataset given in Section 6.2 with the 64 histogram bins

\begin{tabular}{|l|l|l|l|}
\hline Class & $\begin{array}{l}\text { Pedestrian } \\
(\%)\end{array}$ & $\begin{array}{l}\text { Vehicle } \\
(\%)\end{array}$ & $\begin{array}{l}\text { Tree } \\
(\%)\end{array}$ \\
\hline Pedestrian & 91.67 & 6.67 & 1.66 \\
\hline Vehicle & 3.181 & 95.45 & 1.36 \\
\hline Tree & 5 & 3.33 & 91.67 \\
\hline
\end{tabular}

Table 8. Confusion matrix for pedestrian, all types of vehicles and trees as seen in the dataset given in Section 6.2 with the 64 histogram bins

\begin{tabular}{|l|l|l|l|}
\hline Class & $\begin{array}{l}\text { Pedestrian } \\
(\%)\end{array}$ & $\begin{array}{l}\text { Vehicle } \\
(\%)\end{array}$ & $\begin{array}{l}\text { Tree } \\
(\%)\end{array}$ \\
\hline Pedestrian & 95 & 1 & 4 \\
\hline Vehicle & 3.34 & 95.34 & 1.32 \\
\hline Tree & 0.66 & 4.66 & 94.68 \\
\hline
\end{tabular}

Table 9. Confusion matrix for pedestrian, all types of vehicles and trees as seen in the dataset given in Section 6.2 with the 64 histogram bins

\begin{tabular}{|l|l|l|l|}
\hline Class & $\begin{array}{l}\text { Pedestrian } \\
(\%)\end{array}$ & $\begin{array}{l}\text { Vehicle } \\
(\%)\end{array}$ & $\begin{array}{l}\text { Tree } \\
(\%)\end{array}$ \\
\hline Pedestrian & 95.625 & 2.5 & 1.875 \\
\hline Vehicle & 2.245 & 96.11 & 1.645 \\
\hline Tree & 2.13 & 1.39 & 96.48 \\
\hline
\end{tabular}

Table 10. Confusion matrix for pedestrian, all types of vehicles and trees under illumination changes and different poses as seen in the dataset given in Section 6.2 with the 64 histogram bins

\begin{tabular}{|l|l|l|l|}
\hline Class & $\begin{array}{l}\text { Pedestrian } \\
(\%)\end{array}$ & $\begin{array}{l}\text { Vehicle } \\
(\%)\end{array}$ & $\begin{array}{l}\text { Tree } \\
(\%)\end{array}$ \\
\hline Pedestrian & 84.12 & 8.46 & 7.42 \\
\hline Vehicle & 8 & 83.98 & 8.02 \\
\hline Tree & 7.88 & 7.56 & 84.56 \\
\hline
\end{tabular}

This table 7, table 8 , table 9 and table 10 shows that the confusion matrix for the three classes of the evaluation video sets given in the table 1 with 64 histogram bins. The average accuracy for these videos are 92.93, 95.1, 96.07 and 84.22 respectively. The overall performance of the system is 92.08 . 
The Fig. 21 shows the graph which is drawn between the Fmeasure and time at seconds. For each video set mentioned in the table 1, the curve is drawn. In this graph, F-measure value is saturated between 2 and 3 seconds. From the result, the video set above 2 seconds gives better performance of the object identification.

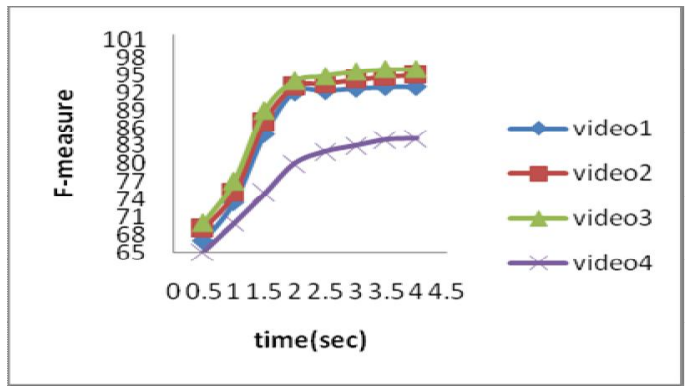

Fig. 21. Performance of the F-measure value for the 4 video sets

\section{CONCLUSION}

In height-width based classification method, background registration technique, segmentation using morphological operator and identification using height and width of objects are presented. The system effectively combines simple domain knowledge about object classes with time domain statistical measures to identify target objects in the presence of partial occlusions. The computational complexity of this algorithm is linear in the size of a video frame and the number of objects detected.

In static-dynamic object classification method, object detection is done without background elimination, segmentation using bounding box registration technique and the classification is done with the multiclass svm. A system has been developed to detect and identify for both dynamic and static objects on highways efficiently. This paper is mainly used to control the increasing traffic-flow on highways and to meet safety and security standards. For Accident avoidance, static object classification is needed with the dynamic object classification. As we have considered traffic on highways there is no question of shadow of any cast such as trees but sometimes due to occlusions two objects are merged together and treated as a single entity.

\section{REFERENCES}

\section{Journal Papers}

[1] Sayanan sivaraman, mohan manubhai trivedi, “A General Active Learning Framework for on-road vehicle Recognition and tracking" in Transactions on Intelligent Transportation systems, vol no 11, issue 2, pages 267272,June 2010 IEEE.

[2] P. M. Daigavane and P. R. Bajaj, " Real Time Vehicle Detection and Counting Method for Unsupervised Traffic Video on Highways", International Journal of Computer Science and Network Security, VOL.10 No.8, pages112117August 2010.

[3]Rajiv Kumar Nath, swapan kumar Deb, “ On Road Vehicle /Object Detection and Tracking using Template", on Indian Journal of Computer Science and Engineering vol. No .2 pages $98-107,2009$.

[4] C. Wang, J. Lien, “Automatic vehicle detection using local features-A statistical approach," IEEE Transactions on
Intelligent Transportation Systems, vol. 9, Issue no. 1, pp. 8396, Mar. 2008.

[5] A.Elgammal R.Duraisamy,D.Harwood and Larry S.Devis , Background \& Foreground modeling using non parametric Kernal density estimation for visual surveillance",on proceeding of the IEEE, vol.90,no 7, pages $1151-1163$ july 2002.

\section{Text Book}

[6] The OpenCV Reference Manual Release 2.4.2 published on july 2012.

\section{Conference Proceedings}

[7]F.Dornaika,san Sebastian, Bilbao, P.Chakik,'Efficient object detection and Matching using Feature classification on Pattern Recognition",20th International Conference on Pattern Recognition(ICPR), 2010 pages 3073 - 3076.

[8] S.Schraml, A.N.Belbachir, N.Brandle,"A Real-Time Pedestrian Classification Method for Event-Based Dynamic Stereo Vision” in IEEE 2010.

[9] G. Jun, J. K. Aggarwal, and M. Gokmen, "Tracking and segmentation of highway vehicles in cluttered and crowded scenes", in IEEE Workshops on Applications of Computer Vision, pages 1-6,2008.

[10] Zhenjun Han, Qixiang Ye, and Jianbin Jiao," Online feature evaluation for object tracking using Kalman Filter" in 19th International Conference on Pattern Recognition, 2008. (ICPR2008), pages 1-4, 2008.

[11] X. Song and R. Nevatia," A model-based vehicle segmentation method for tracking" IEEE Proceedings of the 10th International Conference. on Computer Vision, pages 1124 - 1131 Vol. 22005

[12] N Dalal, B Triggs,"Histograms of Oriented Gradients for Human Detection" in Proceedings of the IEEE Computer Society Conference on Computer Vision and Pattern Recognition 2005. 